\title{
A NOTE \\ TO THE SIERPIŃSKI FIRST CLASS OF FUNCTIONS
}

\author{
Robert Menkyna
}

\begin{abstract}
The purpose of this paper is to establish some theorems concerning approximation and representation of a function of the Sierpiński first class by Darboux function of the Sierpiński first class.
\end{abstract}

In recent years, a number of articles have appeared dealing with a problem of approximating or representing arbitrary real valued functions of real variable by functions with the Darboux (intermediate value) property. In some cases, only functions belonging to a restricted class of functions such as the Baire one class [2, [3], [13] or the class of semicontinuous functions [6], [10], [12] were considered. We deal with the classes of real functions defined on the interval $I=[0,1]$. First, we introduce all relevant notations. Let $C, B_{1}, D, Q$, $S_{s}, l s c$, and $u s c$ stand for the class of continuous, Baire one, Darboux, quasi-continuous, strong Świątkowski, lower and upper semicontinuous functions, respectively. The intersection $D \cap l s c$ will be denoted by $D l s c$, and applying the same principle, we will use notations $D Q l s c, S_{s} l s c$, too. Let $C_{f}$ be a set of all points of continuity of the function $f$, and $D_{f}$ be a set of all points of discontinuity of the function $f$.

A point $x$ is said to be a bilateral c-point of a set $A$ if and only if $(x ; x+\delta) \cap A$ and $(x-\delta ; x) \cap A$ have the cardinality continuum for every $\delta>0$, i.e.,

$$
\operatorname{card}((x ; x+\delta) \cap A)=\operatorname{card}((x-\delta ; x) \cap A)=\mathfrak{c} .
$$

The set $A$ is said to be bilaterally $\mathfrak{c}$-dense in the set $B, B \subset_{\mathfrak{c}} A$, if and only if each point $x \in B$ is a bilateral $\mathfrak{c}$-point of the set $A$.

If a function $f: I \rightarrow \mathbb{R}$ maps connected sets onto connected sets, then it is said to be Darboux.

(c) 2017 Mathematical Institute, Slovak Academy of Sciences.

2010 Mathematics Subject Classification: Primary 26A21, 54C30; Secondary 26A15, $54 \mathrm{C} 08$.

Keyw ords: lower resp. upper semicontinuity, the Darboux property, the strong Światkowski function.

This work was partially supported by the Slovak Grant Agency under the project VEGA No. 1/0676/17. 
Let us recall the notion of quasi-continuity which was introduced by $\mathrm{S}$. K e mp ist y in 1932 [4]. A function $f$ is said to be quasi-continuous at a point $x_{0}$ if for each open set $U \ni x_{0}$ and each open set $V \ni f\left(x_{0}\right)$ there exists a nonempty open set $W \subset U$ such that $f(W) \subset V$.

According to [8], a function $f$ is strong Swiatkowski if whenever $\alpha, \beta \in I$, $\alpha<\beta$ and $y \in(f(\alpha), f(\beta))$, there exists a point $x_{0} \in(\alpha, \beta) \cap C_{f}$ such that $f\left(x_{0}\right)=y$.

Now, we define the Sierpiński first class $S_{1}$ of functions [15]:

$$
S_{1}=\left\{\sum_{n=1}^{\infty} f_{n}: \sum_{n=1}^{\infty}\left|f_{n}(x)\right|<\infty \text { for every } x \in[0,1] \text {, with each } f_{n} \in C\right\} \text {. }
$$

According to [16, the Sierpiński first class coincides with the class of sums of lower semicontinuous and upper semicontinuous functions. For this reason, the Sierpiński first class will be denoted either by $S_{1}$ or, in accordance with [11, by $l s c-l s c$. It is evident that $S_{1} \subset B_{1}$ but $S_{1} \subsetneq B_{1}$ (see [11], [16]). To prove the main results of the paper, we will use of [10, Theorem 3] on approximation of semicontinuous function by Darboux semicontinuous function.

THEOREM 1. Let $f$ be a function such that $f \in l s c$, and let $E$ be an arbitrary $F_{\sigma}$ set which is bilaterally $\mathfrak{c}$-dense in itself. If the set $E$ is bilaterally $\mathfrak{c}$-dense in the set of points of discontinuity of the function $f$, then there exists a function $g \in D l s c$ such that

$$
\begin{array}{ll}
g(x)<f(x) & \text { for } \quad x \in E, \\
g(x)=f(x) & \text { for } \quad x \in I \backslash E .
\end{array}
$$

Now, we prove the theorem on approximation by Darboux function in the Sierpiński first class.

TheOREM 2. Let $f \in S_{1}, f=l+u$, where $l \in l s c$ and $u \in$ usc. If a Borel set $E$ is bilaterally $\mathfrak{c}$-dense in the set of points of discontinuity of functions $l$ and $u$, then there exists a function $g \in D S_{1}$ such that $\{x: f(x) \neq g(x)\} \subset E$.

P r o of. We denote $D^{*}=D_{l} \cup D_{u}$. The set $D^{*}$ is of type $F_{\sigma}$, of first category, that is, $D^{*}=\bigcup_{n=1}^{\infty} D_{n}$, where $D_{1} \subset D_{2} \subset D_{3} \subset \cdots$ are closed nowhere dense sets and, of course, $D_{f} \subset D^{*}$. Additionally, according to [13, Lemma 7], it can be assumed that the set $E$ is of type $F_{\sigma}$, of the first category, bilaterally c-dense in itself and $\bigcup_{n=1}^{\infty} D_{n} \subset_{\mathfrak{c}} E$. Otherwise, the set $E$ can be replaced with its subset having these properties. Thus, let

$$
E=\bigcup_{n=1}^{\infty} F_{n}
$$

where each $F_{n}$ is a nowhere dense closed set. Without loss of generality, it can be 


\section{A NOTE TO THE SIERPIŃSKI FIRST CLASS OF FUNCTIONS}

assumed that $F_{1} \subset F_{2} \subset \cdots$ As $l \in l s c$, there exists a sequence of continuous functions

$$
l_{1}<l_{2}<l_{3}<\cdots
$$

which pointwise converges to the function $l$ and, analogously, as $u \in u s c$, there exists a sequence of continuous functions

$$
u_{1}>u_{2}>u_{3}>\cdots
$$

which pointwise converges to the function $u$. Evidently, the sequence

$$
f_{n}=l_{n}+u_{n}, \quad n \in \mathbb{N},
$$

pointwise converges to the function $f$. Moreover, let $\left\{\varepsilon_{n}\right\}_{n \in \mathbb{N}}$ be a sequence of positive real numbers such that $\varepsilon_{n} \rightarrow 0$. Functions $l_{n}, u_{n}, f_{n}$ are uniformly continuous on $[0,1]$. Thus, the sequence $\left\{\varepsilon_{n}\right\}_{n \in \mathbb{N}}$ determines a sequence of positive numbers $\delta_{n}$ such that for every $x_{1}, x_{2} \in[0,1]$

$$
\begin{aligned}
\left|x_{1}-x_{2}\right|<\delta_{n} \Rightarrow & \left|l_{n}\left(x_{1}\right)-l_{n}\left(x_{2}\right)\right|<\varepsilon_{n}, \\
& \left|u_{n}\left(x_{1}\right)-u_{n}\left(x_{2}\right)\right|<\varepsilon_{n}, \\
& \left|f_{n}\left(x_{1}\right)-f_{n}\left(x_{2}\right)\right|<\varepsilon_{n} .
\end{aligned}
$$

Applying of [9] Lemma 2], we can construct a sequence of perfect sets $P_{n}, n \in \mathbb{N}$, such that

$$
F_{1} \subset \mathfrak{c} P_{1} \subset E
$$

and

$$
\left(F_{n+1} \cup P_{n}\right) \subset_{\mathfrak{c}} P_{n+1} \subset E \quad \text { for every } n \in \mathbb{N} .
$$

It is evident that

$$
P_{1} \subset_{\mathfrak{c}} P_{2} \subset_{\mathfrak{c}} P_{3} \subset_{\mathfrak{c}} \cdots, \quad E=\bigcup_{i=1}^{\infty} P_{i} .
$$

Let for each $n \in \mathbb{N}$ the triple of functions $\left(l_{n}, u_{n}, f_{n}\right)$ be associated with the perfect set $P_{n}$. The set $E$ is $\mathfrak{c}$-dense in $D^{*}$ and therefore $F_{n}$ can be required to satisfy condition

$$
\forall x \in D_{n} \quad \text { there is } \quad a, b \in F_{n} \subset P_{n} \text { such that } a<x<b \wedge b-a<\delta_{n} \text {. (0.2) }
$$

From the existence of a system of perfect sets $P_{n}, n=1,2, \ldots$, it follows that there exists a system of closed sets $P_{\alpha}, \alpha \geq 1$ (proof in [13, Theorem 8]) such that if

$$
\alpha_{1}<\alpha_{2} \Rightarrow P_{\alpha_{1}} \subset_{\mathfrak{c}} P_{\alpha_{2}} \text {. }
$$

Now, for each $i \leq \alpha<i+1$, we define a triple of functions $\left(l_{\alpha}, u_{\alpha}, f_{\alpha}\right)$,

$$
\begin{aligned}
& l_{\alpha}=l_{i}+(\alpha-i)\left(l_{i+1}-l_{i}\right), \\
& u_{\alpha}=u_{i}+(\alpha-i)\left(u_{i+1}-u_{i}\right), \\
& f_{\alpha}=f_{i}+(\alpha-i)\left(f_{i+1}-f_{i}\right),
\end{aligned}
$$

associated with the set $P_{\alpha}$. 
It is easy to see that if

$$
\begin{aligned}
\alpha_{1}<\alpha_{2} \Rightarrow l_{\alpha_{1}} & <l_{\alpha_{2}} \\
u_{\alpha_{1}} & >u_{\alpha_{2}}
\end{aligned}
$$

and

$$
f_{\alpha}=l_{\alpha}+u_{\alpha} \quad \text { for each } \quad \alpha \geq 1 .
$$

Let $\alpha(x)$ have the same sense as in the proof of [10, Theorem 3] if:

$$
x \in E \Rightarrow \alpha(x)=\inf \left\{\alpha: x \in P_{\alpha}\right\} .
$$

Now, we define the functions $l^{*}, u^{*}, f^{*}$ in the way as in the proof in [10, Theorem 3]:

$$
\begin{aligned}
& l^{*}(x)= \begin{cases}l_{\alpha(x)}(x), & \text { if } x \in E, \\
l(x), & \text { if } x \notin E,\end{cases} \\
& u^{*}(x)= \begin{cases}u_{\alpha(x)}(x), & \text { if } x \in E, \\
u(x), & \text { if } x \notin E,\end{cases} \\
& f^{*}(x)= \begin{cases}f_{\alpha(x)}(x), & \text { if } x \in E, \\
f(x), & \text { if } x \notin E .\end{cases}
\end{aligned}
$$

The function $l^{*}$ fulfills identical conditions as the function $g$ in the proof of [10, Theorem 3], that is

$$
\begin{array}{lll}
l^{*} \in D l s c \text { and } & l^{*}<l \text { on } E \\
& l^{*}=l \text { on }[0,1] \backslash E .
\end{array}
$$

A theorem analogous with Theorem 1 can be formulated for the function $u \in u s c$, too. Therefore, the function

$$
\begin{array}{ll}
u^{*} \in D u s c \text { and } \quad & u^{*}>u \text { on } E, \\
& u^{*}=u \text { on }[0,1] \backslash E .
\end{array}
$$

Moreover, the function $f^{*}$ is constructed in the same way as the function $g$ in [13. Theorem 8]. According to the assertion of this theorem,

$$
f^{*} \in D B_{1} \quad \text { and } \quad\left\{x: f^{*}(x) \neq f(x)\right\} \subset E .
$$

Because $f_{\alpha}=l_{\alpha}+u_{\alpha}$ for every $\alpha \geq 1$, from the construction of functions $l^{*}, u^{*}, f^{*}$ it follows that

$$
f^{*}=l^{*}+u^{*} \in S_{1} \subset B_{1} .
$$

Using the last two results, we show that the function

$$
f^{*} \in D B_{1} \cap S_{1}=D S_{1} \quad \text { and for } \quad\left\{x: f^{*}(x) \neq f(x)\right\} \subset E,
$$

that is, the function $f^{*}$ satisfies the assertion of theorem.

The next theorem proves that every Sierpiński one function can be represented as a sum of two Sierpiński one functions with Darboux property. 


\section{A NOTE TO THE SIERPIŃSKI FIRST CLASS OF FUNCTIONS}

THEOREM 3. Let $f$ be a Sierpinski one function on an interval $I, f=l+u$, where $l \in l s c$ and $u \in$ usc. If a Borel set $E \subset C_{l} \cap C_{u}$ is bilaterally $\mathfrak{c}$-dense in the set of points of discontinuity of the functions $l$ and $u$, then there exists a function $g \in D S_{1}$ such that for $\{x: f(x) \neq g(x)\} \subset E$ and the function $f-g \in D S_{1}$.

P r o of. Again, by [13, Lemma 7], there exists a set $E^{*} \subset E \subset C_{f}$ of type $F_{\sigma}$ of the first category, bilaterally $\mathfrak{c}$-dense in itself such that $D_{l} \cup D_{u} \subset_{\mathfrak{c}} E^{*}$. Theorem 2 implies the existence of a function $g \in D S_{1}, g=l^{*}+u^{*}$, where $l^{*} \in l s c$ and $u^{*} \in u s c$ such that the set for $\{x: f(x) \neq g(x)\} \subset E^{*}$. Because the function

$$
f-g=(l+u)-\left(l^{*}+u^{*}\right)=\left(l-u^{*}\right)+\left(u-l^{*}\right) \in S_{1},
$$

it suffices to prove that the function $f-g$ has Darboux property. Let us consider an arbitrary point $x_{0} \in I$.

If $f\left(x_{0}\right)=g\left(x_{0}\right)$, then since the set for $\{x: f(x)=g(x)\}$ is residual in $I$, there exist sequences $x_{n} \nearrow x_{0}, y_{n} \searrow x_{0}, n \in \mathbb{N}, f\left(x_{n}\right)=g\left(x_{n}\right), f\left(y_{n}\right)=g\left(y_{n}\right)$. Thus,

$$
\lim _{n \rightarrow \infty}(f-g)\left(x_{n}\right)=\lim _{n \rightarrow \infty}(f-g)\left(y_{n}\right)=(f-g)\left(x_{0}\right)=0 .
$$

If $f\left(x_{0}\right) \neq g\left(x_{0}\right)$, then $x_{0} \in C_{f}$. The function $g \in D S_{1}$, hence there exist sequences $x_{n} \nearrow x_{0}, y_{n} \searrow x_{0}, n \in \mathbb{N}$, such that

$$
\lim _{n \rightarrow \infty} g\left(x_{n}\right)=\lim _{n \rightarrow \infty} g\left(y_{n}\right)=g\left(x_{0}\right) .
$$

The function $f$ is continuous at the point $x_{0}$. Then,

$$
\lim _{n \rightarrow \infty} f\left(x_{n}\right)=\lim _{n \rightarrow \infty} f\left(y_{n}\right)=f\left(x_{0}\right) .
$$

From the foregoing, it follows that

$$
\lim _{n \rightarrow \infty}(f-g)\left(x_{n}\right)=\lim _{n \rightarrow \infty}(f-g)\left(y_{n}\right)=(f-g)\left(x_{0}\right) .
$$

Following Young in [1], $f-g \in D B_{1}$.

From the above, it follows:

$$
f-g \in D B_{1} \cap S_{1}=D S_{1} \quad \text { and for } \quad\{x ; g(x) \neq f(x)\} \subset E .
$$

Of course, if we choose a first Baire category set $E$ of Lebesque measure zero from Theorem 3, then there exists a Darboux Sierpiński one function $g$ such that

$$
f=g+(f-g), \quad \text { where } \quad f-g \in D S_{1}
$$

and $f-g=0$ except for on the first category measure zero set.

On the other hand, if we do not require validity of $f-g=0$ except for on the measure zero set, then we can show that every Sierpiński one function can be expressed as a sum of two Sierpiński one functions with a stronger property than the Darboux property. Based on results of A. Maliszewski [7], 


\section{ROBERT MENKYNA}

R. M e n k y n a 14] characterized the class of differences of lower semicontinuous strong Swiątkowski functions by equations

$$
(l s c-l s c) \cap D Q=l s c D Q-l s c D Q=S_{s} l s c-\dot{S}_{s} l s c .
$$

Additionally, in [12, it is proved that

$$
l s c=\dot{S}_{s} l s c+\dot{S}_{s} l s c \text { or } u s c=\dot{S}_{s} u s c+\dot{S}_{s} u s c, \quad \text { respectively. }
$$

If we use the validity of the above relations, we can prove the following statement:

THEOREM 4. Let $f$ be a Sierpinski one function on an interval I. Then, there exist Darboux quasi-continuous Sierpinski one functions $f_{1}, f_{2}$ defined on the interval $I$ such that $f=f_{1}+f_{2}$.

P r o of. Because $f$ is the Sierpiński one function, there exists a lower semicontinuous function $l$ and a upper semicontinuous function $u$ such that $f=l+u$. In accord with [12, Theorem 8], there exist lower semicontinuous strong Swiątkowski functions $l_{1}, l_{2}$ and upper semicontinuous strong Świątkowski functions $u_{1}, u_{2}$ such that

$$
l=l_{1}+l_{2} \quad \text { and } \quad u=u_{1}+u_{2} .
$$

Therefore,

$$
f=\left(l_{1}+l_{2}\right)+\left(u_{1}+u_{2}\right)=\left(l_{1}-\left(-u_{1}\right)\right)+\left(l_{2}-\left(-u_{2}\right)\right) .
$$

The function

$f_{1}=l_{1}-\left(-u_{1}\right) \in \dot{S}_{s} l s c-\dot{S}_{s} l s c=l s c D Q-l s c D Q=(l s c-l s c) \cap D Q=D Q S_{1}$

and for the same reason, the function

$$
f_{2}=l_{2}-\left(-u_{2}\right) \in D Q S_{1} .
$$

We have shown that any Sierpiński one function can be written as a sum of two Darboux quasi-continuous Sierpiński one functions.

In addition, our goal is to prove that every Sierpiński one function can be expressed as a sum of two strong Świątkowski functions of Sierpiński one class. By small modifications to the proof of Lemma A.3.2 and to the proof of the implication $i i \Rightarrow$ iii in the proof of [5, Theorem A.3.3], we can prove the folowing lemmas. Naturally, let symbols $\underline{\lim }(f, x), \overline{\lim }(f, x),\|g\|, \omega(f, x)$ have the same meaning as in [5].

LEMMA 5. Assume that $K$ is nowhere dense and closed, the functions $f_{1}, f_{2}, \ldots$ $\ldots, f_{m} \in D Q B_{1}$ are locally bounded on $\mathbb{R} \backslash K$, and $\tau>0$. There is a nowhere dense closed set $F \supset K$ such that $F \backslash K \subset \cap_{j=1}^{m} C_{f_{j}}$, and continuous function $g$, $g=0$ on $K,\|g\| \leq \tau$ such that

$$
\left(f_{j}+g\right)(I \cap F \backslash K) \supset\left[\underline{\lim }\left(f_{j}, x\right), \varlimsup \lim \left(f_{j}, x\right)\right]
$$

for every $x \in K, j=1,2, \ldots, m$ and every open interval $I \ni x$. 


\section{A NOTE TO THE SIERPIŃSKI FIRST CLASS OF FUNCTIONS}

P r o o f. Write $\mathbb{R} \backslash K$ as the union of a sequence of compact intervals $\left\{I_{n}, n \in \mathbb{N}\right\}$ with boundary points from $\cap_{j=1}^{m} C_{f_{j}}$ such that $\operatorname{int}\left(I_{n}\right) \cap \operatorname{int}\left(I_{m}\right)=\emptyset$, for every $n \neq m$. For each $n$, put $c_{n}^{j}=\inf f_{j}\left(I_{n}\right)$ and $L_{n}^{j}=\sup f_{j}\left(I_{n}\right)$. Let $\left\{J_{p}, p \in \mathbb{N}\right\}$ be an enumeration of all open intervals with rational endpoints which intersect $K$. (The lemma is trivial in case $K=\emptyset$.) Fix $p \in \mathbb{N}$. First, observe that for each $k_{0} \in \mathbb{N}, j=1,2, \ldots, m$

$$
\bigcup_{n>k_{0}, I_{n} \subset J_{p}}\left(c_{n}^{j}-\frac{\tau}{2^{p}}, L_{n}^{j}+\frac{\tau}{2^{p}}\right) \supset c l \bigcup_{x \in K \cap J_{p}}\left[\underline{\lim }\left(f_{j}, x\right), \overline{\lim }\left(f_{j}, x\right)\right] .
$$

Indeed, let $y$ belong to the set on the right-hand side of (0.3). There are $x \in K \cap J_{p}$ and $y^{\prime} \in\left[\underline{\lim }\left(f_{j}, x\right), \varlimsup \lim \left(f_{j}, x\right)\right]$ such that $\left|y-y^{\prime}\right|<\frac{\tau}{2^{p+1}}$. Choose $n>k_{0}$ and $x, \in I_{n}$ such that $I_{n} \subset J_{p}$ and $\left|f_{j}\left(x^{\prime}\right)-y^{\prime}\right|<\frac{\tau}{2^{p+1}}$. Then clearly, $y \in$ $\left(c_{n}^{j}-\frac{\tau}{2^{p}}, L_{n}^{j}+\frac{\tau}{2^{p}}\right)$.

Set $n_{0}^{m}=0$. By $(0.3)$, there is $n_{p}^{m}>\cdots>n_{p}^{2}>n_{p}^{1}>n_{p-1}^{m}$ such that

$$
\bigcup_{I_{n} \subset J_{p}, n_{p-1}^{m}<n \leq n_{p}^{1}}\left(c_{n}^{1}-\frac{\tau}{2^{p}}, L_{n}^{1}+\frac{\tau}{2^{p}}\right) \supset \bigcup_{x \in K \cap J_{p}}\left[\underline{\lim }\left(f_{1}, x\right), \varlimsup \lim \left(f_{1}, x\right)\right] \cap[-p, p]
$$

and

$$
\bigcup_{I_{n} \subset J_{p}, n_{p}^{j-1}<n \leq n_{p}^{j}}\left(c_{n}^{j}-\frac{\tau}{2^{p}}, L_{n}^{j}+\frac{\tau}{2^{p}}\right) \supset \bigcup_{x \in K \cap J_{p}}\left[\underline{\lim }\left(f_{j}, x\right), \overline{\lim }\left(f_{j}, x\right)\right] \cap[-p, p]
$$

for $j=2, \ldots, m$. Define $\varepsilon_{n}=\frac{\tau}{2^{p}}$ for $n \in\left\{n_{p}^{1}, \ldots, n_{p}^{m}\right\}$.

For each $n=n_{p}^{j}$, apply [5, Lemma A.3.1] to find a nowhere dense closed set $F_{n} \subset I_{n} \cap \cap_{j=1}^{m} C_{f_{j}}$ and a continuous function $g_{n}$ such that $g_{n}=0$ outside of $I_{n}$, $\left\|g_{n}\right\| \leq 2 \varepsilon_{n}$, and

$$
\left(f_{j}+g_{n}\right)\left(F_{n}\right) \supset\left[c_{n}^{j}-\varepsilon_{n}, L_{n}^{j}+\varepsilon_{n}\right] .
$$

Define $F=K \cup \bigcup_{n \in \mathbb{N}} F_{n}$ and $g=\sum_{n \in \mathbb{N}} g_{n}$. Note that $F$ is nowhere dense and closed, and since $\varepsilon_{n} \rightarrow 0$, so $g$ is continuous.

Let $x \in K, I \ni x$ be an open interval, $j \in\{1,2, \ldots, m\}$ and $y \in\left[\underline{\lim }\left(f_{j}, x\right)\right.$, $\left.\varlimsup \lim \left(f_{j}, x\right)\right]$. There exists $p>|y|$ such that $x \in J_{p} \subset I$. For $n=n_{p}^{j}$, the interval $I_{n} \subset J_{p}$ and $y \in\left(c_{n}^{j}-\frac{\tau}{2^{p}}, L_{n}^{j}+\frac{\tau}{2^{p}}\right)$. Then, $y \in\left(c_{n}^{j}-\varepsilon_{n}, L_{n}^{j}+\varepsilon_{n}\right)$, and by (0.4), there is $t \in F_{n} \subset F$ with $\left(f_{j}+g\right)(t)=y$. The other requirements are easy to prove.

LEMMA 6. Let functions $f_{1}, f_{2}, \ldots, f_{m} \in D Q B_{1}$, and let $\varepsilon>0$ be arbitrary real number. Then, there is a continuous function $g,\|g\| \leq \varepsilon$ such that $f_{j}+g \in S_{s} B_{1}$ for every $j=1,2, \ldots, m$.

P r o of. Set $g_{0}=0$ and $F_{0}=\emptyset$. We will procced by induction. Fix $n \in$ $\mathbb{N}$ and assume that we have defined a closed and nowhere dense set $F_{n-1}$. Define $K_{n}=F_{n-1} \cup \cup_{j=1}^{m}\left\{x \in \mathbb{R}: \omega\left(f_{j}, x\right) \geq n^{-1}\right\}$. Then, $K_{n}$ is closed and 


\section{ROBERT MENKYNA}

nowhere dense. Use Lemma 5 to find a nowhere dense closed set $F_{n} \supset K_{n}$ and a continuous function $g_{n}$ such that

$$
F_{n} \backslash K_{n} \subset \cap_{j=1}^{m} C_{f_{j}}, g_{n}=g_{n-1} \quad \text { on } \quad K_{n},\left\|g_{n}-g_{n-1}\right\| \leq \frac{\varepsilon}{2^{n}},
$$

and

$$
\left(f_{j}+g_{n}\right)\left(I \cap F_{n} \backslash K_{n}\right) \supset\left[\underline{\lim }\left(f_{j}+g_{n-1}, x\right), \varlimsup \overline{\lim }\left(f_{j}+g_{n-1}, x\right)\right]
$$

for every $x \in K_{n}, j=1,2, \ldots, m$ and every open interval $I \ni x$.

Define $g=\lim _{n \rightarrow \infty} g_{n}$ and $h_{j}=f_{j}+g$. Then clearly, $g$ is continuous and $\|g\| \leq \varepsilon$. To prove that $h_{j}$ is strong Świątkowski, we will use [5. Proposition 1.3.18]. Take $x \in D_{h_{j}}=D_{f_{j}}$ and an open interval $I \ni x$. There is $n \in \mathbb{N}$ with $x \in K_{n}$. By (0.5), we obtain

$$
\begin{aligned}
h_{j}\left(I \cap C_{h_{j}}\right) & =h_{j}\left(I \cap C_{f_{j}}\right) \supset h_{j}\left(I \cap F_{n} \backslash K_{n}\right) \\
& =\left(f_{j}+g_{n+1}\right)\left(I \cap F_{n} \backslash K_{n}\right) \supset\left[\underline{\lim }\left(f_{j}+g_{n}, x\right), \overline{\lim }\left(f_{j}+g_{n}, x\right)\right] \\
& =\left[\underline{\lim }\left(h_{j}, x\right), \varlimsup \lim \left(h_{j}, x\right)\right] .
\end{aligned}
$$

(We used that $g_{p}=g_{n+1}$ on $F_{n}$ for $p>n$ and $g_{p}(x)=g_{n}(x)$ for $p \geq n$.) So, by [5, Proposition I.3.18], $h_{j}$ is strong Światkowski.

THEOREM 7. Let $f$ be a Sierpinski one function. Then, there are strong Swiatkowski Sierpinski one functions $g$ and $h$ such that $f=g+h$.

P r o of. If $f$ is a Sierpiński one function on an interval $I$, according to Theorem 4, there exist Darboux quasi-continuous Sierpiński one functions $f_{1}, f_{2}$ defined on the interval $I$ such that $f=f_{1}+f_{2}$. Since $S_{1} \subset B_{1}$, by Lemma 6 , there exists a continuous function $g$ such that $f_{1}+g$ and $-f_{2}+g$ are strong Świątkowski functions. Moreover, because $S_{1}+C=(l s c-l s c)+C=S_{1}$, the functions $f_{1}+g$ and $-f_{2}+g$ are strong Świątkowski Sierpiński one functions. Therefore, the function $f_{2}-g$ is again strong Świątkowski Sierpiński one function, the function $f$ can be represented as a sum of two strong Swiątkowski Sierpiński one functions $f=\left(f_{1}+g\right)+\left(f_{2}-g\right)$.

\section{REFERENCES}

[1] BRUCKNER, A. M.: Differentiation of Real Functions, in: Lecture Notes in Math., Vol. 659, Springer, Berlin, 1978.

[2] BRUCKNER, A. M.-CEDER, J. G.-KESTON, R.: Representations and approximations by Darboux functions in the first class of Baire, Rev. Roum. Math. Pures Appl. 9 (1968), 1247-1254.

[3] GUREVIČ, A. B.: D-continuous Sierpiński components, Dokl. Akad. Nauk BSSR 10 (1966), 539-571. (In Russian)

[4] KEMPISTY, S.: Sur les functions quasicontinues, Fund. Math. 19 (1932), 184-197. 


\section{A NOTE TO THE SIERPIŃSKI FIRST CLASS OF FUNCTIONS}

[5] MALISZEWSKI, A.: Darboux Property and Quasi-Continuity: A Uniform Approach. Wydaw. Uczelniane WSP, 1996.

[6] MALISZEWSKI, A.: On the sums of Darboux upper semicontinuous quasi-continuous functions, Real Anal. Exchange 20 (1994-1995), 244-249.

[7] MALISZEWSKI, A.: On the differences of upper semicontinuous quasi-continuous functions, Math. Slovaca 48 (1998), 245-252.

[8] MALISZEWSKI, A.: On the limits of strong Światkowski functions, Zeszyty Nauk. Politech. Łódz. Mat. 27 (1995), 87-93.

[9] MENKYNA, R.: On approximations of semicontinuous functions by Darboux semicontinuous functions, Real Anal. Exchange 35 (2009-2010), 423-430.

[10] MENKYNA, R.: On the differences of lower semicontinuous functions, Real Anal. Exchange 41 (2015-2016), 123-136.

[11] MENKYNA, R.: On representations of Baire one functions as the sum of lower and upper semicontinuous functions, Real Anal. Exchange 38 (2012-2013), 169-176.

[12] MENKYNA, R.: On the sums of lower semicontinuous strong Swiatkowski functions, Real. Anal. Exchange 39 (2013-2014), 15-32.

[13] MENKYNA, R.-MYDIELKA, L': Approximations by Darboux functions in the Baire one class, Tatra Mt. Math. Publ. 55 (2013), 57-66.

[14] MENKYNA, R.: Difference of two strong Światkowski lower semicontinuous functions, Math. Slovaca 67 (2017), 99-108.

[15] MORAYNE, M.: Sierpiński's hierarchy and locally Lipschitz functions, Fund. Math. 147 (1995), 73-82.

[16] SIERPIŃSKI, W.: Sur les fonctions développables en séries absolument convergentes de fonctions continues, Fund. Math. 2 (1921), 15-27.

Received October 13, 2016

Institute of Aurel Stodola

Faculty of Electrical Engineering

University of Žilina

ul. kpt. J. Nálepku 1390

SK-031-01 Liptovský Mikuláš

SLOVAKIA

E-mail: menkyna@lm.uniza.sk 